\title{
Téoros
}

Revue de recherche en tourisme

\section{La place du tourisme dans l'économie québécoise}

\section{Gabriel Bouchard}

Volume 7, numéro 3, novembre 1988

Économie du tourisme

URI : https://id.erudit.org/iderudit/1080385ar

DOI : https://doi.org/10.7202/1080385ar

Aller au sommaire du numéro

Éditeur(s)

Université du Québec à Montréal

ISSN

0712-8657 (imprimé)

1923-2705 (numérique)

Découvrir la revue

Citer cet article

Bouchard, G. (1988). La place du tourisme dans l'économie québécoise. Téoros, 7(3), 45-48. https://doi.org/10.7202/1080385ar d'utilisation que vous pouvez consulter en ligne.

https://apropos.erudit.org/fr/usagers/politique-dutilisation/ 


\section{La place du \\ tourisme dans \\ l'économie québécoise}

"Le tourisme est si complexe qu'il est malaisé d'en cerner les limites, d'en évaluer l'importance, d'en définir précisément les composantes, d'en analyser les mécanismes et d'en mesurer toutes les implications".

Cette citation, tirée d'un document de travail rédigé par le ministère du Tourisme et intitulé: Le tourisme au Québec - Bilan et perspectives d'action 1985-1988, reflète bien la situation lorsqu ${ }^{4} i s^{4}$ agit de décrire en termes économiques ce secteur d'activité. Le tourisme n'est pas vraiment une industrie bien définie tant il implique des activités et des secteurs différents. Il emprunte à différents secteurs et il ne peut être assimilé en totalité a aucun de ceux reconnus dans le cadre de la comptabilité économique du Québec.
Les donnees, lorsqu'elles sont disponibles, concernent surtout la demande, ce qui rend difficile tout exercice de comparaison avec les autres secteurs de l'économie. Les difficultés au niveau des définitions et des concepts ainsi que les différentes méthodologies utilisées pour recueillir les données statistiques contribuent également à compliquer la situation. Il faut donc tenir compte de ce contexte et manifester une certaine ouverture d'esprit à l'ếgard des données servant à mesurer l'importance économique du tourisme. Celles-ci sont souvent des ordres de grandeur plutôt que des valeurs exactes ou absolues.

En conséquence, le meilleur outil actuellement disponible pour evaluer l'importance Économique du tourisme demeure le modèle intersectoriel développé par le Bureau de la statistique du Québec. C'est un modèle d'analyse de la propagation de la demande dans l'économie québécoise. Il exploite les relations d'échanges en biens et services observées entre les différents secteurs économiques.

Une êtude d'impact économique produite à l'aide du modèle intersectoriel du B.S.Q. donne des résultats exprimés en termes d'effets directs et indirects sur la main-d'oeuvre, les salaires avant impóts, la valeur ajouthe et certaines recettes fiscales et parafiscales. Les effets directs correspondent aux dépenses en salaires et autres revenus bruts des secteurs directement concernés par le tourisme. Les effets indirects se comptabilisent chez les entreprises qui fournissent des. biens et des services aux entreprises touristiques et chez les fournisseurs des fournisseurs.

Cet article reprend donc les donnees d'une simulation produite à l'aide du modèle intersectoriel du B.S.Q.. Les informations recueillies permettront d'établir dans un premier temps, les impacts des dépenses touristiques pour chacune des dimensions liées au tourisme, pour dans un deuxième temps, estimer les effets totaux d'une dépense touristique de 1,005 dans l'économie québécoise en 1987.

Les secteurs reliés au tourisme Les recettes touristiques (elles excluent les dépenses effectuées au cours des excursions de moins de 24 heures) du Québec cuaient de 3228 millions de dollars en 1987 . Elles se répartissent à peu près de la façon suivante entre les principaux secteurs économiques relies au tourisme (voir tableau 1 ).

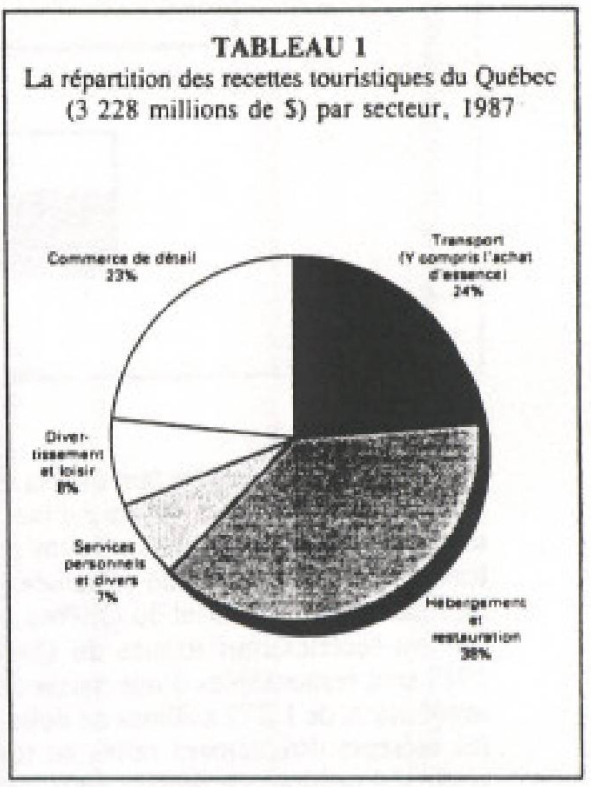

\section{La main-d'oeuvre}

L'impact total de dépenses touristiques de 3228 millions de dollars sur la main-d'oeuvre est d'environ 106000 inputs de maind'oeuvre en personnes-année dont 91000 dans les secteurs directement reliés au tourisme et 15000 dans les autres secteurs. c'est-à-dire chez les fournisseurs.

Cette main-d'oeuvre couvre essentiellement les employés salariés. De plus, il faut noter qu'elle ne correspond pas a l'emploi total mais bien à la charge de travail nécessaire pour soutenir des recettes touristiques de 3228 millions de dollars. À titre d'exemple, trois employés salariés qui travaillent chacun quatre mois comptent pour un seul input de main-d'oeuvre exprime en personnesannéc.

À cause du caractère saisonnier de l'activité touristique, le nombre d'emplois attribuable au tourisme est définitivement plus élevé que 91000 inputs de main-d'oeuvre en personnes-année. A la lumière des données précédentes, on constate qu'il faut des recettes touristiques de l'ordre de 220000 dollars pour le Québec pour soutenir six emplois (en personnes-année) dans les secteurs directement concernés par le tourisme et un chez les fournisseurs (les autres secteurs). 


\section{TABLEAU 2}

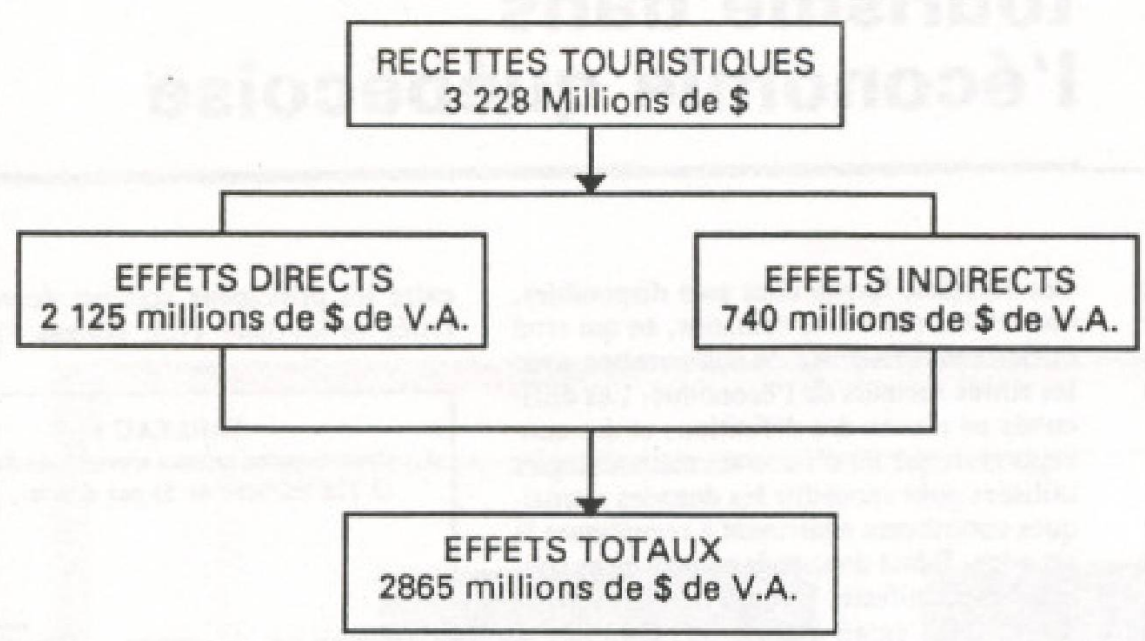

\section{Les salaires}

Les salaires avant impôts tant directs qu"indirects correspondent à la paie brute avant toute déduction et sont des revenus pour les travailleurs. La simulation effectuée à l'aide du modèle intersectoriel du Québec indique que les recettes touristiques du Québec en 1987 sont responsables d'une masse salariale avant impót de 1272 millions de dollars pour les secteurs directement reliês au tourisme et de 354 millions de dollars pour les autres secteurs. En d'autres termes, un dollar de recettes touristiques engendre 0,405 de masse salariale dans les secteurs directement reliés au tourisme et 0,11 dans les autres. Par emploi (personnes-année), ces masses salariales donnent un salaire annuel moyen de $14000 \$$ dans les secteurs directement reliés au tourisme et de $24000 \$$ dans les autres. Ce résultat est une indication que les emplois des secteurs reliés au tourisme sont moins bien rémunérés que les autres.

\section{Les autres revenus}

Les autres revenus avant impóts constituent l'autre composante de la valeur ajoutée au coutt des facteurs. Ils comprennent, entre autres, la rémunération de l'entrepreneur, la rémunération du capital (amortissement, épuisement et dépréciation des bătiments et du matériel), les intérêts divers (intérêt sur la dette: hypothécaire, obligataire, etc.) et les autres frais y compris les charges patronales et les bénéfices marginaux.

La simulation produite à l'aide du modèle indique, pour 1987, que ces autres revenus s'élevaient à 853 millions de dollars dans les secteurs dits touristiques et à 384 millions de dollars dans les autres secteurs. L'addition des effets tant directs qu'indirects donnent 1237 millions de dollars d'autres revenus bruts avant impóts.

\section{La valeur ajoutée}

$\mathrm{La}$ valeur ajoutee ou la valeur ajoutee au coût des facteurs représente la rémunération des facteurs de production à l'intérieur de l'économie québécoise. C'est la somme des postes salaires avant impôts et autres revenus bruts avant impöts. On peut également la calculer en déduisant la valeur des achats en biens et services (auprès des fournisseurs) des dépenses d'exploitation totales. Les deux façons de la calculer donnent sensiblement les mémes résultats. Le produit intérieur brut au coût des facteurs de production est une autre expression pour désigner la valeur ajoutée.

Les 3228 millions de dollars de recettes touristiques en 1987 au Québec ont permis de créer approximativement 2125 millions de dollars de valeur ajoutée directement dans les secteurs concernés par le tourisme et 740 millions de dollars dans les autres secteurs (chez les fournisseurs).

La valeur ajoutée directement attribuable au tourisme ( 2125 millions de dollars) est légèrement supérieure à celle du secteur agricole (2 079 millions de dollars) en 1987. Si on compare cette valeur ajoutée à celle engendrée par les principaux secteurs manufacturiers, on observe que seulement six de ceux= ci créent plus de valeur ajoutée que le tourisme en termes absolus en 1987 (voir les tableaux 2 et 3 ).

Cette simulation permet également d'observer que la valeur ajoutée par emploi (personnes-année) dans les secteurs directement reliés au tourisme est de $23000 \mathrm{dol}$ lars (effets directs) comparativement à 49000 dollars pour celle des secteurs non directement reliés au tourisme (effets indirects).
TABLEAU 3

La valeur ajoutée par le tourisme eomparée a celle des principaux secteurs manufacturiers du Québec, 1987

\begin{tabular}{lc}
\hline Secteurs manufacturiers & $\begin{array}{c}\text { Valeur ajoutéte } \\
\text { (en millions (5)) }\end{array}$ \\
\hline 1 - Aliments et boissons & 3388 \\
2 - Papiers et produits connexes & 3328 \\
3 - Produits chimiques & 2408 \\
4 - Première transformation & 2376 \\
de métaux & 2246 \\
5 - Matériel de transport & 2172 \\
6 - Produits électriques et & 2125 \\
$\begin{array}{l}\text { Tólectroniques } \\
\text { Tourisme }\end{array}$ & 1947 \\
8 - Habillement & 1891 \\
9 - Produits métalliques & 1831 \\
\hline industries connexes &
\end{tabular}

N.B.: Ces données, sauf celles sur le tourisme, proviennent de la publication suivante: La situation Économique au Québec, Bureau de la statistique du Quebec, Edition 1988.

\section{Les revenus pour le gouvernement du Québec}

Les résultats fournis par le modèle permettent d'evaluer certaines recettes fiscales et parafiscales directes et indirectes attribuables aux recettes touristiques. Les recettes pour le gouvernement du Québec comprennent les revenus des taxes indirectes québécoises (taxe de vente québécoise, taxes sur l'essence, les repas, le tabac, les télécommunications, les paris mutuels ainsi que sur la bière, les vins et les spiritueux), l'impót sur le revenu et la parafiscalité québécoise. Cette dernière comprend les contributions de l'employeur et de l'employé à la Régie des rentes du Québec et les contributions de l'employeur à la Régie de l'assurancemaladie du Québec et à la Commission de la santé et de la sécurité au travail du Québec.

Le tourisme constitue une source de revenu importante pour les différents gouvernéments, surtout celui du Québec. Au total, les 3228 millions de dollars de recettes touristiques en 1987 ont généré 328 millions de dollars pour le gouvernement du Québec dont 242 millions de dollars proviennent des secteurs directement concernés par le tourisme et 86 millions de dollars des autres secteurs. Ces 328 millions de dollars représentent $1,6 \%$ de tous les revenus du gouvernement du Québec. Nous n'avons pas considéré pour les fins du calcul les revenus du gouvernement du Québec provenant des sociétés d'État et des transferts du gouvernement du Québec provenant des sociétés d'État et des transferts du gouvernement du Canada. Ce ratio tombe a $1.2 \%$ lorsqu'on considère uniquement les rentrées fiscales attribuables aux seuls secteurs touristiques.

Sous un autre angle, on peut dire qu'un dollar de recettes touristiques génère des ren- 


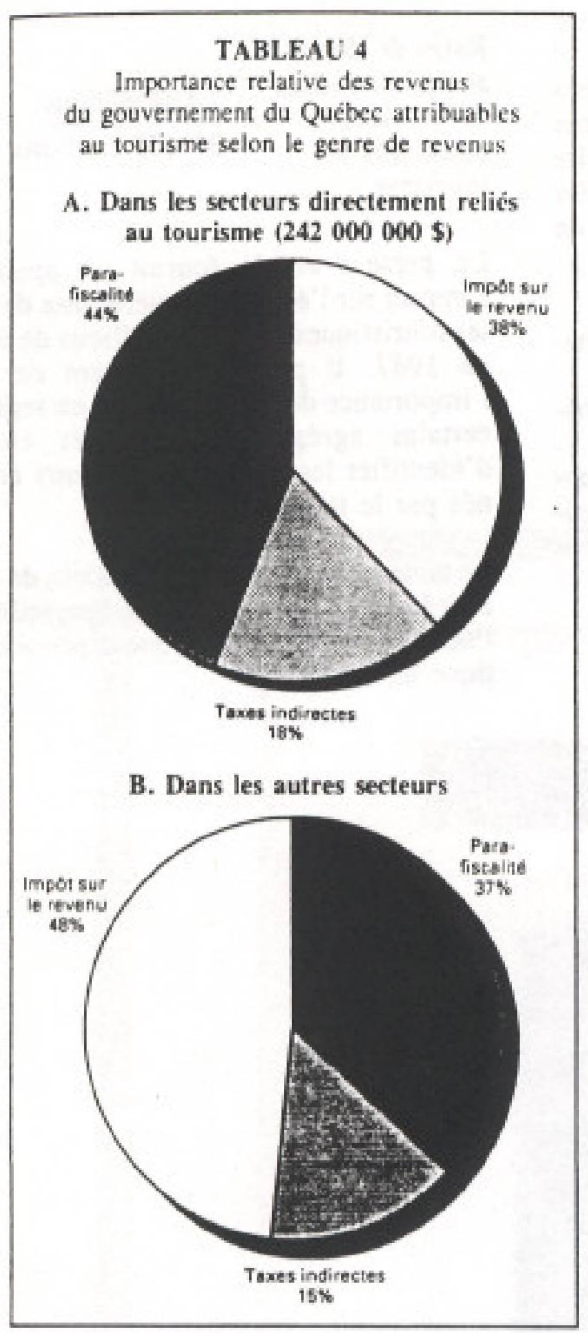

trées fiscales totales de 0,105 pour le gouvernement du Québec dont sept cents et demie proviennent des secteurs directement concernes par le tourisme. Dans les secteurs directements concernés par le tourisme, la figure suivante (voir tableau 4) indique que la parafiscalité devance l"impôt sur le revenu comme source de rentrées fiscales pour le gouvernement du Québec. On observe l'inverse dans les secteurs non directement concernés par le tourisme, l'impôt sur le revenu étant la première source de rentrées fiscales. On peut expliquer cette situation par le fait que les salaires versés dans les industries reliées au tourisme sont moins élevés que ceux versés dans les autres secteurs. Ils génèrent donc moins de revenus pour le gouvernement du Québec sous forme d"impôt sur le revenu.

\section{Les revenus pour le gouvernement du Canada}

Les revenus du gouvernement du Canada comprennent l'impót fédéral sur le revenu, les taxes fédérales indirectes et la parafiscaliné fédérale, laquelle inclut les cotisations de l'employeur et de l'employé à la Commisison d'assurance-chomage.

Au total, les recettes touristiques du Québec ont généré 199 millions de dollars de reve-

TABLEAU 5

La propagation dans l'économic québecoise d'une dépense touristique de 1,00 \$ (en 1987)

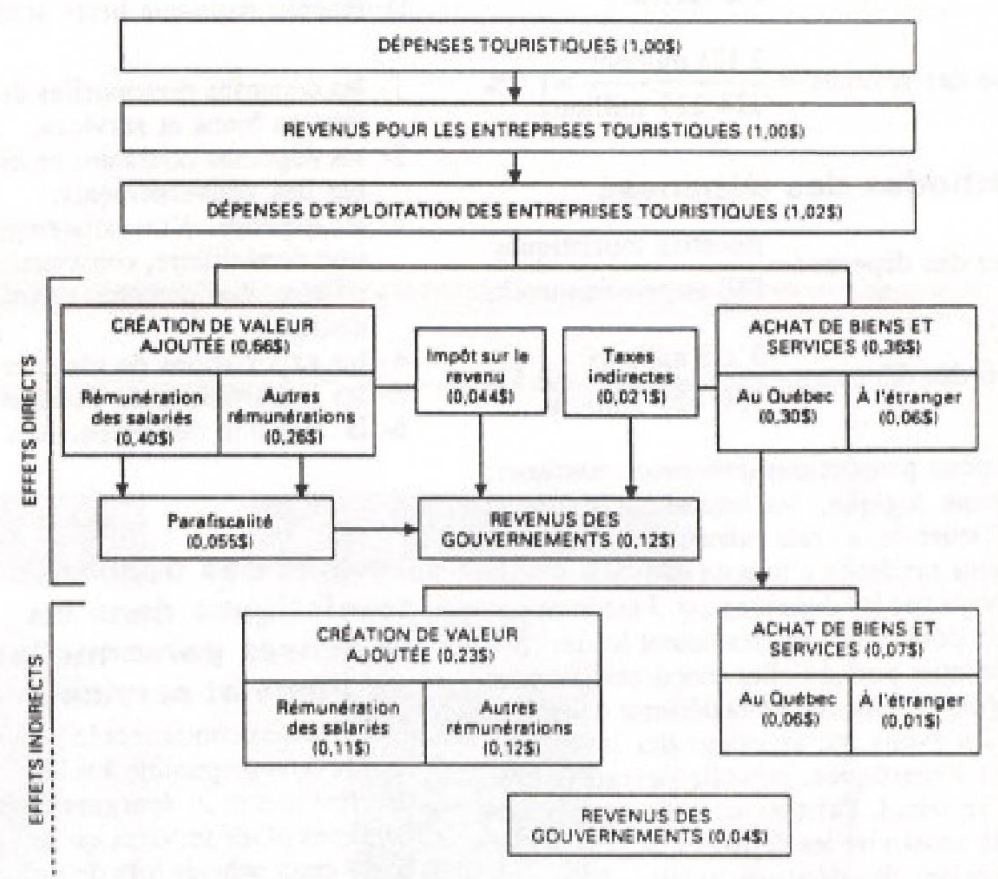

nus pour le gouvernement du Canada dont 145 millions de dollars proviennent des secteurs directement relies au tourisme et 54 millions de dollars des autres secteurs. Un dollar de recettes touristiques au Québec génère des rentrées fiscales de $0,06 \$$ pour le gouvernement du Canada comparativement a $0,10 \$$ pour celui du Québec.

Enfin, il est à noter que les données concernant les rentrées fiscales pour les gouvernements du Québec et du Canada sont légèrement sous-estimés car le modele intersectoriel ne tient pas compte des impóts sur les bénéfices des sociétés.

\section{Le tourisme et les exportations}

Les recettes touristiques du Québec au titre du tourisme international s'élevaient à 808 millions de dollars en 1986 . Elles représentaient $4 \%$ de la valeur totale des exportations internationales du Québec, lesquelles totalisaient 19982 millions de dollars. Si on compare ces recettes touristiques aux principaux produits d'exportation internationale du Québec, on constate qu"elles arrivent au quatrieme rang soit juste en avant les exportations de bois d'oeuvre, mais loin en arrière des exportations concernant les produits suivants:
- le papier d'impression (2 935 millions de dollars);

- les automobiles et les châssis (1 914 millions de dollars):

- l'aluminium et les alliages (1 505 millions de dollars).

\section{Le tourisme et les importations}

Les dépenses des Québécois au chapitre du tourisme international s'élevaient à 1271 millions de dollars en 1986. Elles représentaient $5,9 \%$ de la valeur totale des importations internationales du Québec, lesquelles se chiffraient a 21436 millions de dollars. C'est un ratio relativement élewé qui tend à démontrer que le tourisme constitue un secteur important de fuites (sortie de devises) pour le Québec. D'ailleurs, seules les importations concernant les produits automobiles et chàssis devancent le tourisme à ce chapitre au Québec ( 3180 millions de dollars en 1986).

\section{Le tourisme et le P.I.B.}

Il est possible d'estimer que la part du tourisme dans le produit intérieur brut quebécois en 1987 se situait entre $1,8 \%$ et $2,5 \%$ selon qu'elle est évaluée par la méthode des revenus (valeur ajoutée, P.I.B. au coût des facteurs) ou par celles des dépenses. 
Méthodes des revenus

Ratio des revenus $=\frac{\begin{array}{l}\text { Valeur ajoutée } \\ \text { par le tourisme } \\ \text { (effets directs) }\end{array}}{\begin{array}{l}\text { P.I.B. au coùts } \\ \text { des facteurs }\end{array}}$

Ratio des revenus $=\frac{2125 \text { millions }}{114227 \text { millions }}=1.9 \%$

\section{Méthodes des dépenses}

Ratio des dépenses $=\frac{\text { Recettes touristiques }}{\text { PIB au prix du marché }}$ Ratio des dépenses $=\frac{3228 \text { millions }}{130421 \text { millions }}=2.5 \%$

Ces deux proportions devraient constituer, en toute logique, les limites inférieure et supérieure de la vraie valeur. Toutefois, une certaine prudence s"impose dans le cas de la méthode par les dépenses car il est impossible de désagréger suffisamment les recettes touristiques pour qu'elles concordent avec les composantes mêmes de la dépense nationale. Nous n'avons pas la valeur des investissements touristiques, laquelle devrait figurer dans le calcul. Par contre, il n'est pas possible de soustraire les dépenses de voyage des entreprises des dépenses totales. II ne faudrait pas que celles-ci soient prises en compte dans le calcul.

Pour bien saisir toute la signification des données apparaissant dans cette section-ci, il est nécessaire de rappeler certains concepts de comptabilité nationale.

\section{Produit intérieur brut au prix du marché}

$C^{\prime}$ est la somme de tous les biens et services produits sur un territoire au cours d'une période de temps, ceux-ci étant exprimés en dollars courants. On peut le calculer de deux façons différentes, à savoif:

1- par la méthode des revenus crés au cours du processus de production (méthode de la valeur ajoutée);

2- par la méthode des dépenses nationales.

\section{Produit intérieur brut au coût des facteurs de production}

C'est la somme de toutes les rémunérations des facteurs de production qui sont intervenus au cours du processus de production des biens et services. Les composantes du produit intérieur brut au coùt des facteurs sont:

1- la rémunération des salariés avant impôts et autres déductions;

2- les profits des sociétés avant impóts:

3- les intéréts et revenus divers de pla. cement:

4- le revenu net des exploitants agricoles;

5- le revenu net des entreprises non agricoles, non constituées en société.

\section{Dépense nationale brute}

C'est la somme de toutes les dépenses effectuées pour se procurer les biens et services résultant d'une production finale, c'est-à-dire les biens et services qui en sont au dernier stade de transformation. Les composantes de la dépense nationale brute sont:

1- les dépenses personnelles de consommation en biens et services;

2 - les dépenses courantes en biens et services des gouvernements;

3- les dépenses d'investissement (construction domiciliaire, construction non domiciliaire, équipement et matériel de production):

4- les exportations de biens et services;

5- les importations de biens et services;

6- la variation des inventaires.

\section{La part des dépenses touristiques dans les dépenses personnelles en biens et services}

Les ménages consacrent la majeure partie de leur revenu disponible a acheter des biens et des services et ils épargnent le reste. Parmi les biens et les services qu'ils se procurent, il $y$ a ceux achetés lors de voyages touristiques (au Québec ou à l'étranger). C'est ce qu'on appelle les dépenses touristiques. La part des dépenses personnelles en biens et services de consommation des Québécois consacrée au tourisme était de $3,4 \%$ en 1986.

Ratio des
dépenses $=$

touristiques Dépenses personnelles en biens et services de consommation

$\begin{aligned} & \text { Ratio des } \\ & \text { dépenses } \\ & \text { touristiques }\end{aligned}=\frac{2459 \text { millions }}{71674 \text { millions }}=3.4 \%$

Pour calculer ce ratio, il faut enlever la partie des dépenses touristiques qui n'est pas à proprement dite des dépenses personnelles. Il s'agit des dépenses touristiques qui ont été payées par d'autres agents économiques. $\mathrm{Ce}$ sont les dépenses concernant les voyages d'affaires et les congrès et qui sont généralement payées par les gouvernements, les entreprises ou les associations. On estime à environ $30 \%$ la part de celles-ci dans les dépenses totales consacrées au tourisme, soit 1050 millions de dollars. Comme le tourisme est fortement concentré dans les secteurs des services, il est aussi intéressant de connaître la part des dépenses personnelles en services consacrée au tourisme. Ce ratio était de $7,8 \%$ en 1986 .

Ratio des dépenses personnelles en services consacrés au $=\frac{\text { Dépenses touristiques }}{\text { Dépenses en services }}$ tourisme
Ratio de dépenses personnelles en services consacrés au $=\frac{2459 \text { millions }}{31673 \text { millions }}=7.8 \%$ tourisme

Le présent article fournit un aperçu de l'impact sur l'économie québécoise de recettes touristiques de 3228 millions de dollars en 1987. Il permet également de situer l'importance de cette industrie en regard de certains agrégats économiques en plus d'identifier les principaux secteurs concernés par le tourisme.

Le tableau 5 permet, en conclusion, de visualiser les effets globaux de la propagation dans l'économie québécoise d'une dépense touristique de $1,00 \$$. f 